\title{
Association between aminotransferase/ alanine aminotransferase ratio and cardiovascular disease mortality in patients on peritoneal dialysis: a multi-center retrospective study
}

Xiaoran Feng ${ }^{1 \dagger}$, Yueqiang Wen $^{2 \dagger}$, Fen Fen Peng ${ }^{3}$, Niansong Wang ${ }^{4}$, Xiaojiang Zhan ${ }^{5^{*}}$ and Xianfeng Wu ${ }^{4^{*}}$

\begin{abstract}
Background: Elevated aspartate aminotransferase/alanine aminotransferase (AST/ALT) ratio is an independent risk factor for cardiovascular disease (CVD) among the general population. However, an association between AST/ALT ratio and CVD mortality in patients on peritoneal dialysis (PD) has received little attention.

Methods: A total of 2224 incident PD patients from multi-centers were enrolled from November 1, 2005, to June 30, 2017, in this retrospective cohort study. The primary endpoint was CVD mortality. Eligible patients were divided into high and normal groups according to the AST/ALT ratio cut-off for CVD mortality with the receiver operating characteristic (ROC) curve. The associations between the AST/ALT ratio and CVD mortality were evaluated by the Cox regression model.

Results: Of eligible 1579 patients with a mean age of $49.3 \pm 14.6$ years, $55.4 \%$ of patients were male, $18.1 \%$ of patients had diabetes, and $64.2 \%$ of patients had hypertension. The prevalence of a high AST/ALT ratio was $76.6 \%$ in the cohort population. During a follow-up period with 4659.6 patient-years, 316 patients died, of which 193 (61.1\%) deaths were caused by CVD episodes. The incidence of CVD mortality in the high group was significantly higher than that in the normal group $(13.1 \%$ versus $9.2 \%, P=0.024)$. Cumulative CVD mortality rates were significantly different between the two groups by Kaplan-Meier analysis [hazards ratio $(\mathrm{HR})=1.50$, 95\% confidence index $(\mathrm{Cl})$ 1.092.07, $P=0.014]$. After adjusting for confounding factors, a higher AST/ALT ratio was independently associated with an increased risk of CVD mortality compared with their counterparts ( $H R=1.43,95 \% \mathrm{Cl} 1.08-2.41, P=0.002)$.
\end{abstract}

Conclusions: PD patients with high baseline AST/ALT ratio levels may be at a significant risk of CVD mortality.

Keywords: Aspartate aminotransferase/alanine aminotransferase ratio, Cardiovascular disease, Mortality, Peritoneal dialysis

\footnotetext{
*Correspondence: zhanxiaogang87@163.com; xianfengwu2@163.com

${ }^{+}$Xiaoran Feng and Yuegiang Wen are contributed equally to this work.

${ }^{5}$ Department of Nephrology, The First Affiliated Hospital of Nanchang

University, No. 17, Yongwaizheng Street, Donghu District, Nanchang City

330006, Jiangxi province, China

${ }^{4}$ Department of Nephrology, Affiliated Sixth People's Hospital, Shanghai Jiao

Tong University, No.600, Yi Shan Road, Shanghai, China

Full list of author information is available at the end of the article
}

C C The Author(s). 2020 Open Access This article is licensed under a Creative Commons Attribution 4.0 International License, which permits use, sharing, adaptation, distribution and reproduction in any medium or format, as long as you give appropriate credit to the original author(s) and the source, provide a link to the Creative Commons licence, and indicate if changes were made. The images or other third party material in this article are included in the article's Creative Commons licence, unless indicated otherwise in a credit line to the material. If material is not included in the article's Creative Commons licence and your intended use is not permitted by statutory regulation or exceeds the permitted use, you will need to obtain permission directly from the copyright holder. To view a copy of this licence, visit http://creativecommons.org/licenses/by/4.0/. The Creative Commons Public Domain Dedication waiver (http://creativecommons.org/publicdomain/zero/1.0/) applies to the data made available in this article, unless otherwise stated in a credit line to the data. 


\section{Background}

Cardiovascular disease (CVD) represents the leading cause of death in peritoneal dialysis (PD) patients, accounting for up to $40-60 \%$ of deaths $[1,2]$. Traditional risk factors, such as diabetes, hypertension, dyslipidemia, and a history of CVD, account for up to 50\% of CVD in dialysis patients. At the same time, renal specific markers, including anemia, disordered bone mineral metabolism, and oxidative stress, also likely contribute to the total CVD burden in these patients [3-7]. Therefore, exploring new non-traditional risk factors for CVD episodes may be beneficial to further improve the prognosis of PD patients.

Alanine aminotransferase (ALT) is only found in the liver, but aspartate aminotransferase (AST) in the liver and heart tissue [8]. Compared to ALT, AST significantly increased in patients with CVD events, presenting an elevated AST/ALT ratio of those patients $[9,10]$. As compared to patients with a normal AST/ALT ratio, those with a high AST/ALT ratio had a higher preexisting CVD rate, suggesting that the increased AST / ALT ratio may implicit heart load and injury and potential CVD episodes $[11,12]$. Among the general population participating in a community-based health screening with a 10-year follow-up, the increased AST/ ALT ratio had an independent association with CVD mortality, considering to be a new non-traditional risk for CVD episodes [8]. Another study from the United Kingdom showed that elevated AST/ALT ratio is significantly associated with an increased risk of developing CVD in men with no history of CVD at baseline [13]. It was noteworthy that patients on dialysis had reduced serum levels of aminotransferases [14, 15], whereas whether the AST/ALT ratio was an independent predictor of CVD mortality in dialysis patients remains unknown. In the present study, the aim of this study was to evaluate the association between the AST/ALT ratio and CVD mortality in PD patients.

\section{Methods}

\section{Study design and population}

A retrospective cohort study were conducted, with incident PD patients between November 1, 2005, and February 28, 2017, from four PD centers. Participants with aged less than 18 years at the start of PD or $<$ three PD vintage were excluded by the end of study. The AST/ ALT ratio can increase by alcohol consumption and cardio-hepatic interaction [16, 17]. Patients with preexisting CVD or liver disease are at significant risk of CVD episodes. Thus, to minimize the effect of a history of CVD or liver disease on the association between AST/ALT ratio and CVD mortality, we excluded those with pre-existing CVD or liver disease. To further minimize selective bias, we excluded those with an AST/
ALT ratio $>2$, who may have underlying liver disease. Thus, patients were excluded from the study if they had current drinking, had been diagnosed with a history of CVD, chronic liver disease, or AST or AST values more than two times higher normal values. The study was approved by the Human Ethics Committee of each research center, consistent with the ethical principles of the Declaration of Helsinki. Eligible participants signed a informed consent.

Two experienced investigators at each center recorded demographic characteristics, laboratory variables, medical records and prescriptions at baseline, such as age, sex, body mass index (BMI) diabetes, hypertension, hyperlipidemia, gastrointestinal bleeding, Charlson comorbidity index (CCI), and current smoking. Ejection fraction, estimated glomerular filtration rate (eGFR), hemoglobin, serum albumin, AST, ALT, total bilirubin, cholesterol, triglycerides, high-density lipoprotein (HDL), low-density lipoprotein (LDL), high-sensitivity $\mathrm{C}$-reactive protein (hs-CRP), N-terminal -prohormone BNP (NT-proBNP), and 24-h urine output at baseline were included.

CVD mortality and all-cause mortality were mainly interesting points. The lethal causes were evaluated by two experienced nephrologists at each center. Participants transferring to hemodialysis with less than three-month survival were thought not to be censored, indicating PD treatment failed. Participants were followed up until PD cessation, death, or May 31, 2017. Transferring to hemodialysis (for more than three months), transferring to other centers, renal transplantation, loss of follow-up, or still survival with a follow-up duration of 5 years were considered to be censored. Eligible participants were conducted PD schedules in the light of International Standardized Peritoneal Dialysis Guidelines.

\section{Definitions}

CVD is defined as coronary heart disease, arrhythmias, sudden death, congestive heart failure, or cerebrovascular disease [18]. Chronic liver disease is defined as hepatitis B or C, alcoholic and non-alcoholic liver disease, and autoimmune liver disease [13, 19, 20]. We defined aminotransferase elevation as any value above normal of ALT or AST based on a recent, nationally representative the United States survey AST > $40 \mathrm{IU} / \mathrm{L}$, or ALT > 43 IU/L) [21]. The comorbidity score was calculated using CCI [22]. eGFR was calculated using the Chronic Kidney Disease Epidemiology Collaboration equation [23].

\section{Statistical analysis}

AST and ALT measured according to the standard measurements of Chinese. Data were expressed as mean \pm standard deviation, percentages, or median (25th-75th percentile). All eligible patients were divided into high 
and normal groups according to the AST/ALT ratio cutoff for CVD mortality with the receiver operating characteristic (ROC) curve. Baseline variable comparisons between groups were conducted using t-tests, MannWhitney test, or $\chi^{2}$ analyses. The association between baseline variables and high AST/ALT ratio was analyzed using Logistic regression. Variables clinically considered to be associated with high AST/ALT ratio were picked into a multivariate-adjusted Logistic regression model. Survival analysis was undergone by the Kaplan-Meier curve, alongside with the log-rank test. The hazard ratio (HR) of the AST/ALT ratio for CVD and all-cause mortality were analyzed using Cox regression. Crude HR was first examined (Model 1), followed by adjusting for age, sex, CCI, current smoking, and medication use (Model 2). In the next Model 3, BMI, eGFR, hemoglobin, albumin, total bilirubin, cholesterol, triglycerides, hs-CRP, and 24-h urine output were enrolled to assess whether the AST/ALT ratio had an independent association with interesting points, independent of confounding factors. $P<0.05$ was considered statistically significant. Statistical analysis was performed using GraphPad software 8.0 (GraphPad Prism Software Inc., San Diego, California) and $\mathrm{R}$ software package 3.6.0 (https://www.r-project.org/).

\section{Results}

\section{Baseline characteristics}

A total of 2224 incident PD patients were enrolled in the present study, of whom ten patients younger than 18 years, 84 patients on $\mathrm{PD}<3$ months, 35 with current drinking, 279 with a history of CVD, 134 with chronic liver disease, 77 without baseline AST/ALT ratio, and 26 with AST or ALT values $\geq$ two times higher than normal values were excluded. The remaining 1579 patients with baseline AST/ALT ratio were eligible for the present analysis (Fig. 1). Of 1579 patients with CCI of $3.87 \pm$ 1.66 , the mean age was $49.3 \pm 14.6$ years, $55.4 \%$ were male sex, $18.1 \%$ had diabetes, $64.2 \%$ had hypertension, and $16.2 \%$ had hyperlipidemia.

In ROC curve analysis (supplement), the AST/ALT ratio (area under curve $=0.75,95 \%$ CI $0.72-0.77 ; p<0.001$ ) was found to be a significant predictor of CVD mortality with a sensitivity (79.4\%) and specificity (75.8\%). The cut-off of the AST/ALT ratio for CVD mortality was 1.0 in the cohort population. A total of 1210 (76.6\%) patients were in the high group and $369(23.4 \%)$ patients in the normal group. The baseline characteristics of the study population are shown in Table 1. Patients with high AST/ALT ratio were older $(P<0.001)$, likely to be female sex $(\mathrm{P}<0.001)$, had higher frequency of hyperlipidemia $(P=0.029)$ and statin use $(P=0.037)$, had higher $\mathrm{CCI}(\mathrm{P}<0.001)$ and LDL $(P=0.038)$, and had a lower ALT values $(P<0.001)$ as compared to their counterparts.

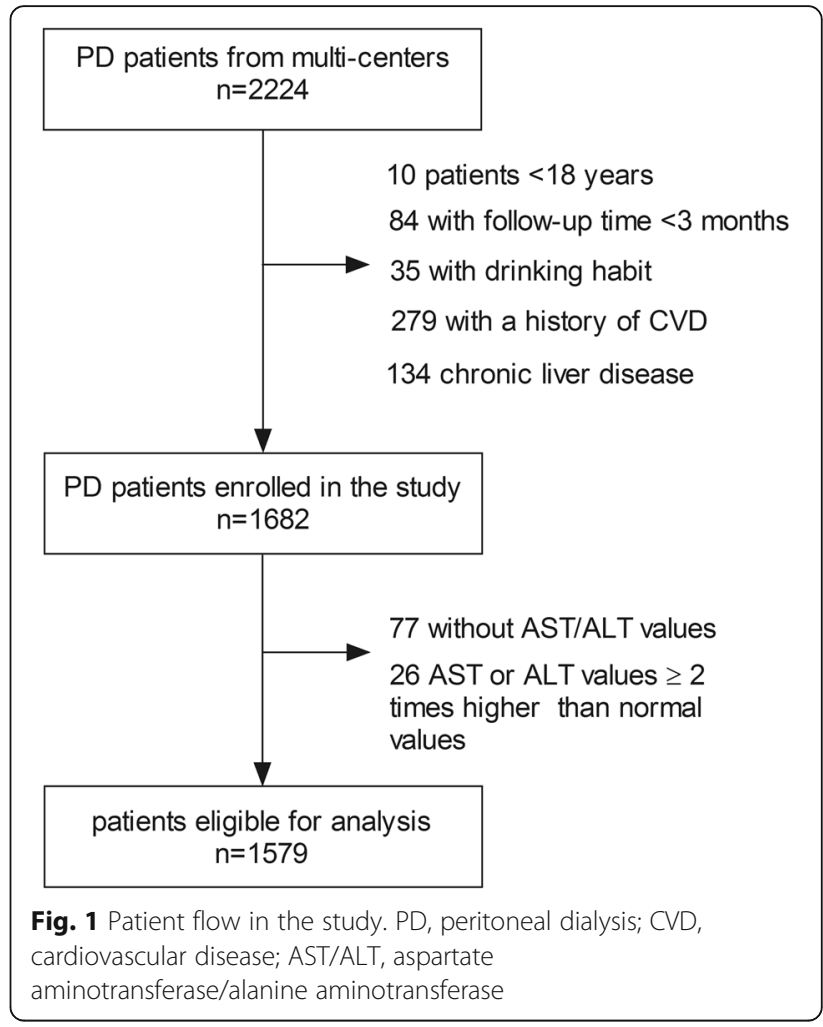

\section{The high AST/ALT ratio}

The prevalence of the high AST/ALT ratio was $76.6 \%$ (74.5-78.7\%) in the cohort population (Fig. 2). Multivariate Logistic analysis showed that older age [increased pre one year, $\mathrm{HR}=1.02,95 \%$ confidence interval $(\mathrm{CI})$ $1.01-1.03, P<0.001]$ and female $(\mathrm{HR}=2.94,95 \% \mathrm{CI}$ 2.30-3.77, $P<0.001)$, statin using $(\mathrm{HR}=1.66,95 \% \mathrm{CI}$ $1.22-2.44, P=0.011)$, and lower bilirubin $(\mathrm{HR}=0.54$, 95\%CI $0.33-0.90, P=0.018)$ were independently associated with the high AST/ALT ratio (Table 2).

\section{Baseline AST/ALT ratio and endpoints}

The total follow-up period was 4659.6 patient-years. By the end of this study, 316 (20.0\%) patients had died, 106 (6.7\%) patients had undergone renal transplantation, 247 (15.6\%) patients had transferred to hemodialysis, 18 (1.1\%) patients had transferred to other PD centers, and 60 (3.8\%) patients had been lost to follow-up; the remaining $832(52.7 \%)$ patients were still followed at these PD centers. Of 316 deaths, 193 (61.1\%) deaths were caused by CVD episodes. The Kaplan-Meier estimates showed that the cumulative CVD and all-cause mortality incidence were significantly different between two AST/ALT ratio groups $(\mathrm{HR}=1.50,95 \% \mathrm{CI} 1.09$ 2.07, and $\mathrm{HR}=1.53,95 \% \mathrm{CI} 1.16-1.93$, Fig. 3a and Fig. 4a). At the end of 1, 3, and 5 years in this study, the incidence of CVD mortality was $8.1,15.8$, and $24.5 \%$ in the high group, and 6.1, 10.2, and $15.2 \%$ in the normal 
Table 1 Baseline characteristics of patients stratified by baseline AST/ALT ratio

\begin{tabular}{|c|c|c|c|c|}
\hline Variables & cohort $(n=1579)$ & Normal group $(n=369)$ & High group $(n=1210)$ & $P$ value \\
\hline Age (years) & $49.3 \pm 14.6$ & $45.8 \pm 13.7$ & $50.3 \pm 14.7$ & $<0.001$ \\
\hline Male (\%) & $874(55.4)$ & $276(74.8)$ & $598(49.4)$ & $<0.001$ \\
\hline $\mathrm{CCl}$ & $3.87 \pm 1.66$ & $3.59 \pm 1.58$ & $3.95 \pm 1.68$ & $<0.001$ \\
\hline Diabetes (\%) & $286(18.1)$ & $64(17.3)$ & $222(18.3)$ & 0.700 \\
\hline Hypertension (\%) & $1014(64.2)$ & $244(66.1)$ & $770(63.6)$ & 0.420 \\
\hline Hyperlipidemia (\%) & $256(16.2)$ & $46(12.5)$ & $210(17.4)$ & 0.029 \\
\hline Gastrointestinal bleeding (\%) & $41(2.6)$ & $10(2.7)$ & $31(2.6)$ & 0.853 \\
\hline Current smoking (\%) & $47(3.0)$ & $6(1.6)$ & $41(3.4)$ & 0.113 \\
\hline ACEI/ARB use (\%) & $518(32.8)$ & $114(30.9)$ & $404(33.4)$ & 0.410 \\
\hline Calcium antagonist use (\%) & $1141(72.3)$ & $272(73.7)$ & $869(1.8)$ & 0.507 \\
\hline$\beta$-blocker use (\%) & $505(32.0)$ & $122(33.1)$ & $383(31.7)$ & 0.611 \\
\hline Diuretic use (\%) & $94(6.0)$ & $20(5.4)$ & $74(6.1)$ & 0.707 \\
\hline Statin use (\%) & $157(9.9)$ & $26(7.0)$ & $131(10.8)$ & 0.037 \\
\hline BMI $\left(\mathrm{kg} / \mathrm{m}^{2}\right)$ & $22.1 \pm 3.6$ & $22.3 \pm 4.5$ & $22.0 \pm 3.3$ & 0.305 \\
\hline Ejection fraction (\%) & $60.0 \pm 5.9$ & $59.8 \pm 6.6$ & $60.0 \pm 5.7$ & 0.820 \\
\hline $\mathrm{eGFR}\left(\mathrm{ml} / \mathrm{min} / 1.73 \mathrm{~m}^{2}\right)$ & $5.63(4.38-8.47)$ & $5.81(4.31-8.93)$ & $5.50(4.01-8.64)$ & 0.367 \\
\hline Hemoglobin (g/dL) & $8.6 \pm 2.0$ & $8.6 \pm 2.1$ & $8.6 \pm 2.0$ & 0.873 \\
\hline Albumin (g/dL) & $3.5 \pm 0.5$ & $3.5 \pm 0.5$ & $3.4 \pm 0.5$ & 0.059 \\
\hline AST (IU/L) & $18(14-23)$ & $19(13-25)$ & $18(14-23)$ & 0.086 \\
\hline ALT (IU/L) & $13(8-20)$ & $36(27-56)$ & $8(5-11)$ & $<0.001$ \\
\hline Bilirubin (mg/dL) & $0.30(0.22-0.41)$ & $0.33(0.27-0.40)$ & $0.30(0.21-0.41)$ & 0.565 \\
\hline Cholesterol (mg/dL) & $159 \pm 60$ & $156 \pm 55$ & $160 \pm 61$ & 0.361 \\
\hline Triglyceride (mg/dL) & $132 \pm 97$ & $126 \pm 90$ & $133 \pm 98$ & 0.198 \\
\hline $\mathrm{HDL}(\mathrm{mg} / \mathrm{dL})$ & $45.4 \pm 16.3$ & $44.7 \pm 17.1$ & $45.6 \pm 16.1$ & 0.432 \\
\hline LDL (mg/dL) & $99.0 \pm 38.8$ & $95.1 \pm 37.0$ & $100.2 \pm 39.3$ & 0.038 \\
\hline hs-CRP (mg/L) & $4.03(1.97-11.04)$ & $2.25(0.85-19.5)$ & $4.25(2.04-14.20)$ & 0.209 \\
\hline NT-pro-BNP (pg/mL) & 2017 (800-6545) & $771(412-4835)$ & $2375(840-5760)$ & 0.824 \\
\hline 24-h urine output (mL) & $851 \pm 534$ & $890 \pm 589$ & $839 \pm 527$ & 0.131 \\
\hline
\end{tabular}

AST/ALT aspartate aminotransferase/alanine aminotransferase, CCI Charlson comorbidity index, ACEI/ARB angiotensin-converting enzyme inhibitor/angiotensin receptor blocker, $B M I$ body mass index, eGFR estimated glomerular filtration rate, $H D L$ high-density lipoprotein, $L D L$ low-density lipoprotein, high-sensitivity $C$ reactive protein ( $h$ s-CRP), NT-pro-BNP N-terminal -prohormone BNP

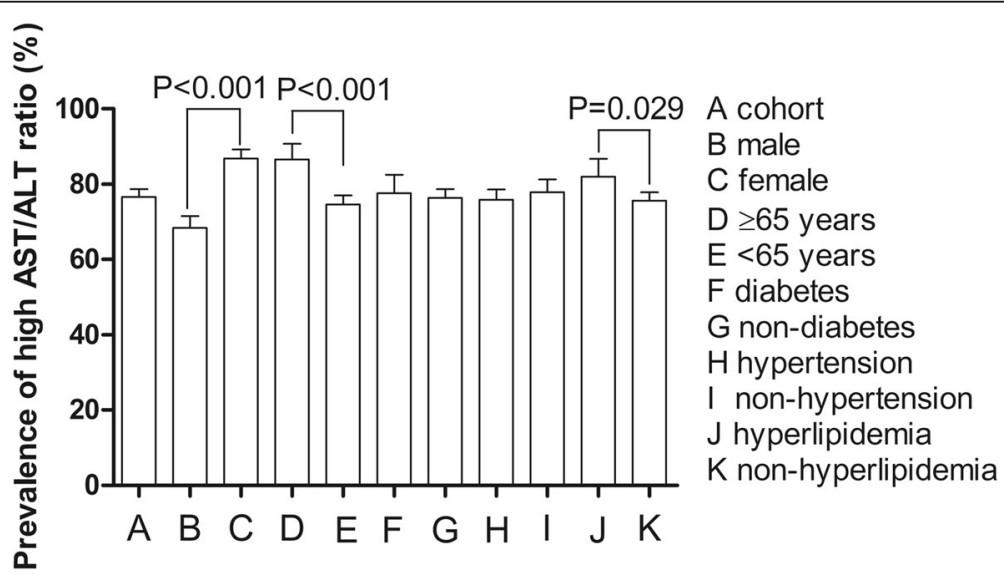

Fig. 2 Prevalence of the high AST/ALT ratio in the cohort population and subgroups. AST/ALT, aspartate aminotransferase/alanine aminotransferase 
Table 2 Predictors for high AST/ALT ratio by Logistic regression

\begin{tabular}{lll}
\hline Variables & \multicolumn{2}{l}{ Multivariate Logistic regression } \\
\cline { 2 - 3 } & HR $(95 \% \mathrm{Cl})$ & $P$ value \\
\hline Age (increased pre 1 years) & $1.02(1.01-1.03)$ & $<0.001$ \\
Female (yes/no) & $2.94(2.30-3.77)$ & $<0.001$ \\
Current smoking (yes/no) & - & - \\
CCl (increased per 1 score) & - & - \\
Hypertension (yes/no) & - & - \\
Hyperlipidemia (yes/no) & - & - \\
Statin use (yes/no) & $1.66(1.12-2.44)$ & 0.011 \\
Bilirubin (increased per 1 mg/dL) & $0.54(0.33-0.90)$ & 0.018 \\
\hline
\end{tabular}

Variables clinically considered to be associated with high AST/ALT ratio were picked into a multivariate-adjusted Logistic regression model. AST/ALT aspartate aminotransferase/alanine aminotransferase, CCI Charlson comorbidity index, LDL low-density lipoprotein

group, respectively. The incidence of all-cause mortality was $12.7,26.7$, and $32.6 \%$ in the normal group, and 9.8, 17.8 , and $20.7 \%$ in the high group, respectively.

The association between the baseline AST/ALT ratio and CVD and all-cause mortality is shown in Table 3. Crude Cox model analysis showed that a high AST/ALT ratio was associated with an increased risk of CVD and all-cause mortality $(\mathrm{HR}=1.63,95 \% \mathrm{CI} 1.13-2.27$; $\mathrm{HR}=$ 1.58, 95\%CI 1.18-2.10, Model 1). Multivariate Cox model analysis found that patients with a high AST/ALT ratio carried a higher risk of CVD and all-cause mortality $(\mathrm{HR}=1.43,95 \%$ CI $1.08-2.41$, and $\mathrm{HR}=1.45,95 \% \mathrm{CI}$ 1.13-2.37, Model 3), even after adjusting for confounding factors. In addition, the association between quartiles of AST/ALT ratio and CVD mortality were shown in Table 4.

\section{Subgroup analyses}

The prevalence of high AST/ALT ratio ranged from $68.4 \%$ (95\%CI $65.3-71.5 \%)$ to $86.9 \%$ (95\%CI 84.3-89.3\%) among all subgroups (Fig. 2). Survival analysis showed that the cumulative CVD mortality incidence between high and normal groups was a significant difference in the male and non-hyperlipidemia subgroups (Fig. $3 \mathrm{~b}$ and c). The cumulative all-cause mortality incidence between high and normal groups was a significant difference in the non-diabetes, hypertension, and nonhyperlipidemia subgroups (Fig. 4 b, c, and d). Adjusted HRs for CVD mortality were conducted in the male and non-hyperlipidemia subgroups, and for all-cause mortality in the non-diabetes, hypertension, and nonhyperlipidemia subgroups by the Cox regression models (Fig. 5).

\section{Discussion}

In the present study, we found that higher baseline AST/ALT ratio may carry an increased risk of CVD and

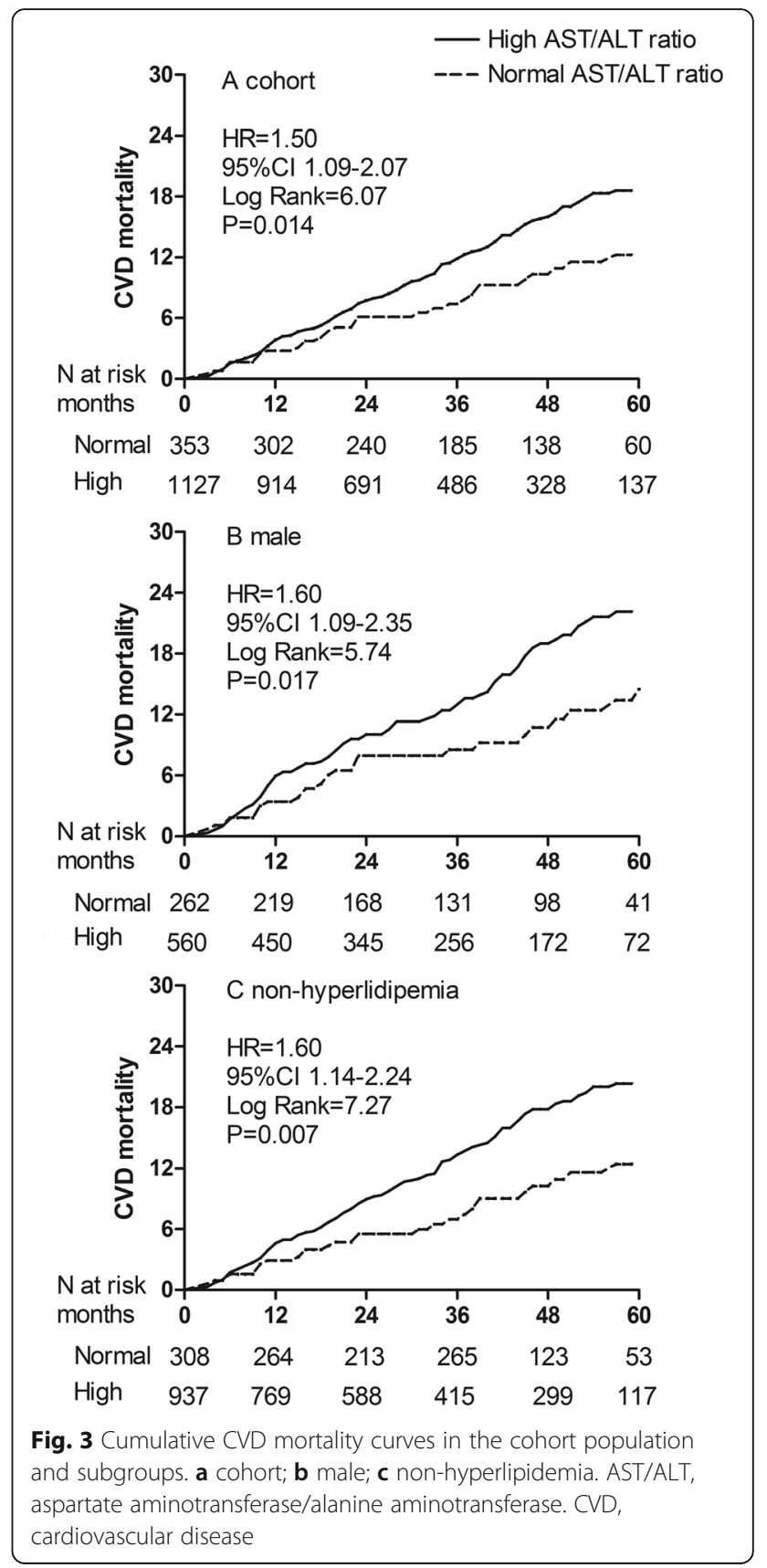

all-cause mortality in PD patients. Also, even though we excluded those patients with chronic liver disease, or a history of CVD, PD patients at the commencement of PD may have a higher prevalence of high AST/ALT ratio. PD patents have high risk of CVD episodes and liver disease, suggesting patients may have unknown underlying cardiovascular disease or liver disease. We should cautiously explain the association between AST/ALT ratio and CVD mortality.

Aminotransferase, including AST and ALT, is a wellknown marker for liver injury. AST is in both the liver and myocardial tissue, but ALT is only in the liver [8]. 


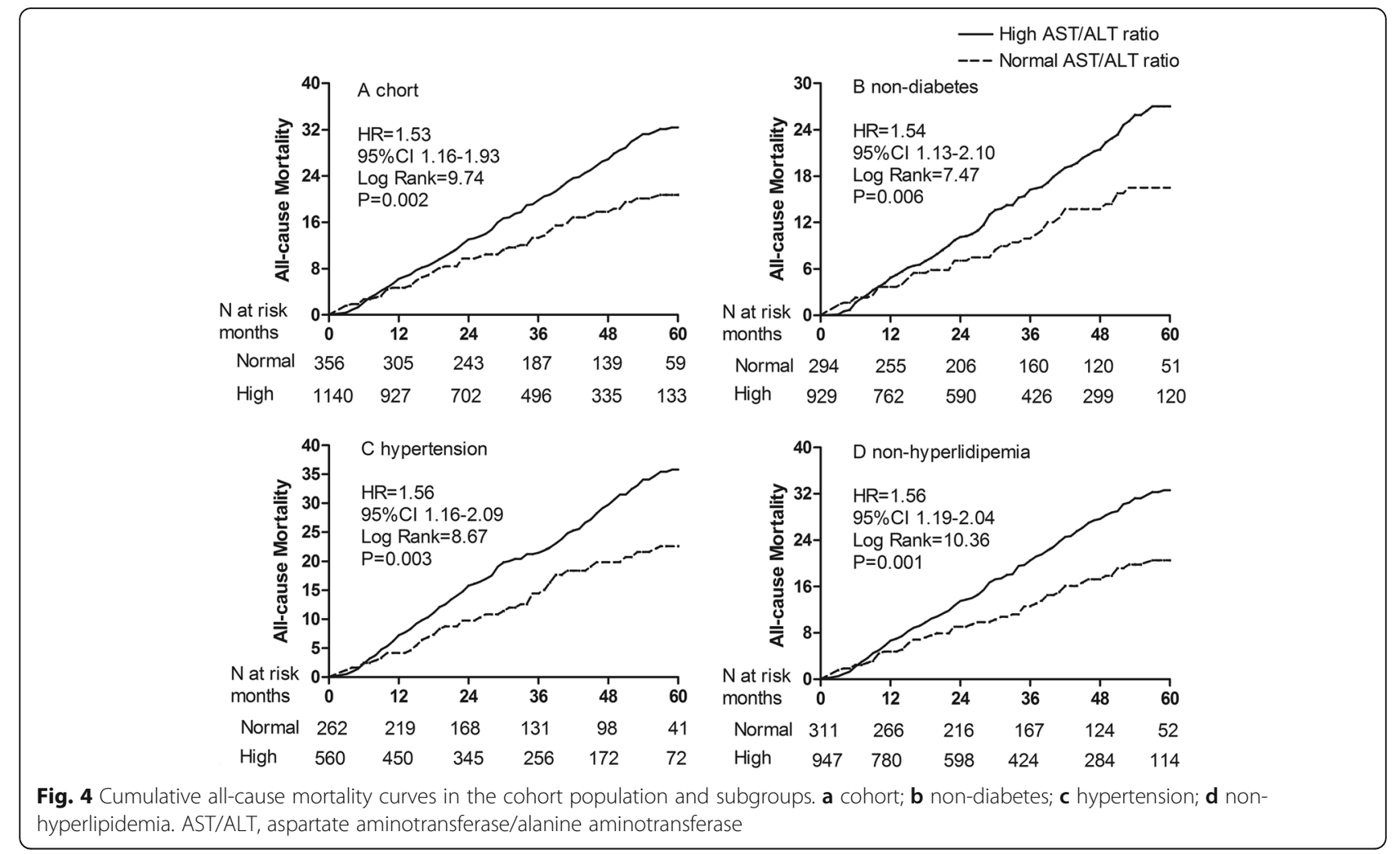

The elevation of the AST/ALT ratio is due to induction by alcohol consumption and cardio-hepatic interaction $[16,24]$. As compared to participants with a lower AST/ ALT ratio, those with a high AST/ALT ratio had a higher pre-existing CVD prevalence, indicating a higher AST/ALT ratio may implicit heart damage and overload, as well as underlying CVD episodes [8]. The Japanese study of 3494 participants more than 40 years found that the high AST/ALT ratio had an independent association with all-cause and CVD mortality, with a 10-year followup [8]. In this study, participants with end-stage renal disease, incomplete data, or study withdrawal were excluded, but those with alcohol habits or pre-existing CVD were not excluded, suggesting less convincing of

Table 3 Adjusted hazards ratio for CVD and all-cause mortality using Cox regression models

\begin{tabular}{llll}
\hline & Model 1 & Model 2 & Model 3 \\
& HR (95\%) & HR (95\%) & HR (95\%) \\
\hline CVD mortality & $1.63(1.13-2.27)$ & $1.55(1.10-2.36)$ & $1.43(1.08-2.41)$ \\
All-cause mortality & $1.58(1.18-2.10)$ & $1.48(1.16-2.24)$ & $1.45(1.13-2.37)$ \\
\hline
\end{tabular}

Hazards ratio: high AST/ALT ratio vs. normal AST/ALT ratio. Model 1: unadjusted. Model 2: adjusted for age, sex, $\mathrm{CCl}$, smoking, and medication use. Model 3: model 2 adjusted for BMI, eGFR, hemoglobin, albumin, bilirubin, cholesterol, triglycerides, hs-CRP, and 24-h urine output

AST/ALT aspartate aminotransferase/alanine aminotransferase, CVD cardiovascular disease, $\mathrm{CCl}$ Charlson comorbidity index, BMI body mass index, eGFR estimated glomerular filtration rate, high-sensitivity C-reactive protein (hs-CRP) their findings due to selective bias. The Italy 6-year follow-up study with 2529 diabetes showed that the AST/ALT ratio had an independent association with allcause and CVD mortality. Patients with a known history of drug-induced liver injury, viral hepatitis, cirrhosis of any etiology, and hemochromatosis were also excluded, but those with a history of CVD failed to be excluded in this study [25]. More recently, a 10-year follow-up prospective study of 29,316 participants aged between 25 and 84 years from the United Kingdom showed that an increased AST/ALT ratio were at significant risk of developing CVD episodes in male participants but not

Table 4 The association between quartiles of AST/ALT ratio and CVD mortality

\begin{tabular}{llll}
\hline & $\begin{array}{l}\text { Model 1 } \\
\text { HR (95\%) }\end{array}$ & Model 2 & Model 3 \\
& HR (95\%) & HR (95\%) \\
\hline Quartiles 1 & Reference & & \\
Quartiles 2 & $1.77(1.24-2.51)$ & $1.40(0.96-2.05)$ & $1.39(0.96-2.03)$ \\
Quartiles 3 & $1.73(1.20-2.50)$ & $1.70(1.20-2.41)$ & $1.71(1.21-2.43)$ \\
Quartiles 4 & $1.67(1.16-2.41)$ & $1.58(1.10-2.27)$ & $1.49(1.20-2.18)$ \\
\hline
\end{tabular}

Model 1: unadjusted. Model 2: adjusted for age, sex, $\mathrm{CCl}$, smoking, and medication use. Model 3: model 2 adjusted for BMI, eGFR, hemoglobin, albumin, bilirubin, cholesterol, triglycerides, hs-CRP, and 24-h urine output AST/ALT aspartate aminotransferase/alanine aminotransferase, CVD cardiovascular disease, $\mathrm{CCl}$ Charlson comorbidity index, BMI body mass index, eGFR estimated glomerular filtration rate; high-sensitivity C-reactive protein (hs-CRP) 


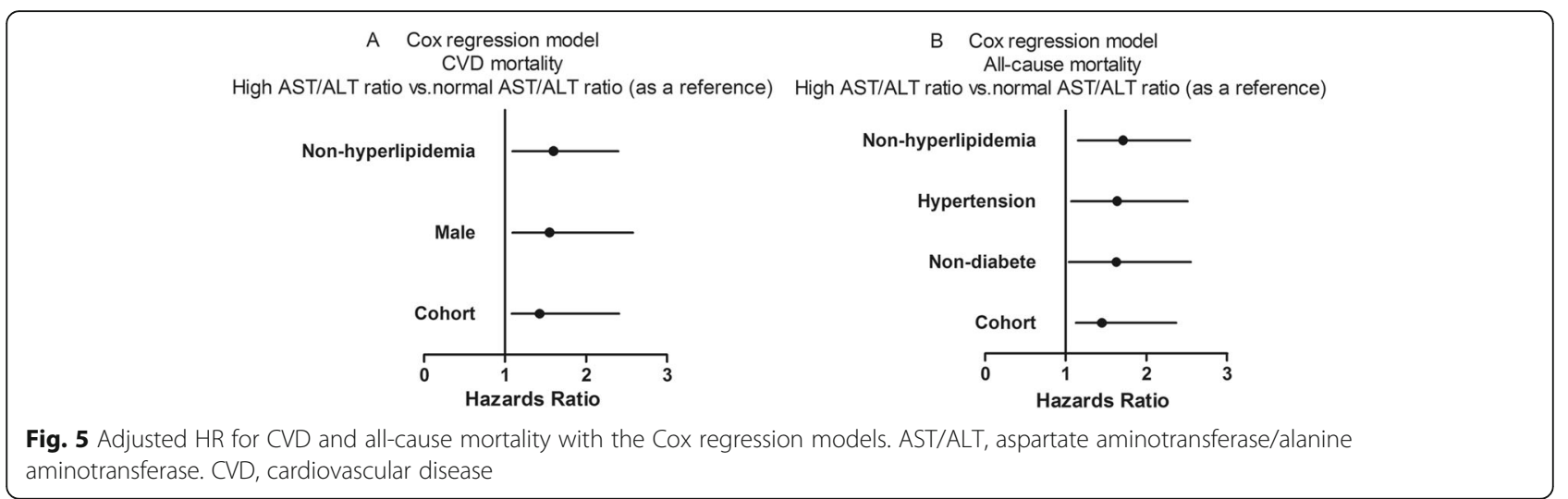

those female, with no baseline pre-existing CVD [13]. Nonetheless, when added to the traditional CVD prediction tools such as Framingham Risk Scores, the AST/ ALT ratio did not contribute to any extra benefits in predicting the CVD accuracy. The major limitation was that patients with chronic liver disease were not excluded from this study. In the present study, to reduce selection bias, we excluded those current drinking, liver disease, and those with a history of CVD. We found that a higher AST/ALT ratio was independently associated with an increased risk for CVD and all-cause mortality. PD patients with a high AST/ALT ratio may have a 1.43-fold higher risk of CVD mortality and a 1.45 -fold higher risk of all-cause mortality compared with their counterparts, even after adjustment for confounding factors. Subgroup analyses showed that a high AST/ALT ratio remained an independent predictor for CVD mortality in those male and non-hyperlipidemias, and allcause mortality in those non-diabetes, hypertension, and non-hyperlipidemias. These findings suggested, along with previous studies, that PD patients with a higher AST/ALT ratio may have more CVD and all-cause involvement, and a preprocedural AST/ALT ratio, a widely available and inexpensive biomarker, might be helpful for risk stratification of CVD and all-cause mortality in PD patients.

A previous study reported that the prevalence of high AST/ALT ratio $\geq 1.0$ was $37.9 \%$ in 2529 type 2 diabetes patients with a 6-year follow-up [13]. Patients with chronic liver diseases were excluded, but those with a history of CVD were not excluded from this study, which may lead to an over-estimated prevalence of high AST/ALT ratio. To date, the prevalence of the high AST/ALT ratio in dialysis patients has received little attention. In the present study, we excluded those with chronic liver disease or a history of CVD, which may be considered as an essential reason to increase the AST/ ALT ratio. Nonetheless, the prevalence of a high AST/ ALT ratio was $76.6 \%$ in the cohort study and ranged from 68.4 to $86.9 \%$ among all subgroups. Thus, there might be a higher prevalence of high AST/ALT ratio in PD patients. These findings suggested, along with previous studies, that future studies should further investigate the prevalence of the AST/ALT ratio in dialysis patients and whether the prognosis of PD patients might be improved by the management of the high AST/ALT ratio.

ALT has potential value as a novel biomarker of aging [13]. Decreased ALT resulted from a reduced liver size and liver blood flow and was associated with aging, frailty, and higher mortality in the general elderly population [26, 27]. There is a correlation between ALT levels and the severity of renal failure [15]. Patients on dialysis had reduced serum levels of aminotransferases, which suggested that the ALT levels were reduced concomitantly with the progression of renal dysfunction $[14,15]$. Previous study demonstrated that ALT levels have a negative correlation with the severity of renal failure, resulting in high AST/ALT ratio in PD patients. In the present study, The ALT levels were reduced concomitantly with the progression of renal dysfunction, resulting in high prevalence of AST/ALT in PD patients. Thus, whether management of AST/ALT ratio can improve the prognosis of PD patients is worth further evaluation.

This study has some limitations. First, we retrospectively reported the independent association, but not causal relationships, between the AST/ALT ratio and the interesting points. We can not adjust all confounding factors of CVD and all-cause mortality, and not eliminated completely the residual confounding effect. Nonetheless, given the effect of residual confounding on the interesting outcomes, we adjusted for potential risk factors using multiply regression analysis. Second, PD patients usually took multiple drugs simultaneously due to other complications. So, it was difficult to determine which drugs may influence liver aminotransferase because of the interaction of drugs. Although we failed to exclude those patients whose liver aminotransferase may influence by multiple drugs, those with AST or ALT values $\geq$ two times higher than normal values were 
excluded. Thus, the effect of drugs on liver aminotransferase may be minimized. Third, rare chronic diseases such as hemochromatosis, which may influence aminotransferase activity, failed to be excluded in the present study. Fourth, we only evaluated baseline variables rather than changes over time in these variables of CVD and all-cause mortality. Additionally, With the development of PD technique, medications, and the treatment of other complications, the prognosis of PD patients have been dramatically improved. Thus, changes over time may affect the results of PD patients, suggesting those receiving more advanced composited managements may gain more benefits than those ante-counterparts. Finally, because PD patients were all Chinese in the present study, the results may not apply to other ethnic PD patients.

\section{Conclusions}

In conclusion, a high AST/ALT ratio at the initiation of PD was independently associated with an increased risk for CVD and all-cause mortality in PD patients. In addition, there may be a higher prevalence of high baseline AST/ALT ratio in PD patients. AST/ALT ratio, as a risk factor of non-traditional CVD mortality, should be monitored regularly in PD patients, reminding us of the evaluation of CVD episodes promptly. Fortunately, since laboratory assays for the AST/ALT ratio are common, readily available, and inexpensive, the AST/ALT ratio could be a promising parameter to identify PD patients at high risk for CVD and all-cause mortality. Future research should further investigate the prevalence of the AST/ALT ratio in PD patients and prospectively evaluate whether the prognosis of PD patients may be improved by the management of the AST/ALT ratio.

\section{Supplementary information}

Supplementary information accompanies this paper at https://doi.org/10. 1186/s12882-020-01840-7.

\section{Additional file 1.}

\section{Abbreviations \\ AST/ALT: Aspartate aminotransferase/alanine aminotransferase; CVD: Cardiovascular disease; CCl: Charlson comorbidity index; ACEI/ ARB: Angiotensin-converting enzyme inhibitor/angiotensin receptor blocker; BMI: Body mass index; eGFR: Estimated glomerular filtration rate; HDL: High- density lipoprotein; LDL: Low-density lipoprotein; hs-CRP: High-sensitivity C- reactive protein; NT-pro-BNP: N-terminal -prohormone BNP}

\section{Acknowledgements}

We express our gratitude to all patients who participated in the study.

\section{Authors' contributions}

XR F, contributions to the conception, interpretation of data, and drafted the work; FF P, the acquisition, analysis and interpretation of data; YQ W, the acquisition, analysis and interpretation of data; NS W, contributions to the conception and design of the work; $X J Z$, contributions to the conception and design of the work; XF W, contributions to the conception, design of the work, and revised it. All authors have read and approved the manuscript.

Funding

No.

\section{Availability of data and materials}

The datasets used and/or analyzed during the current study are available from the corresponding author on reasonable request.

\section{Ethics approval and consent to participate}

The study was consistent with the ethical principles of the Declaration of Helsinki and was approved by the Human Ethics Committee of the Second Affiliated Hospital of Guangzhou Medical University, Zhujiang Hospital of Southern Medical University, Jiujiang No. 1 People's Hospital, Affiliated Sixth People's Hospital, Shanghai Jiao Tong University, and the First Affiliated Hospital of Nanchang University. Written informed consent was obtained from all participants.

Consent for publication

Not Applicable.

\section{Competing interests}

The authors declare that they have no competing interests.

\section{Author details}

'Department of Nephrology, Jiujiang No. 1 People's Hospital, Jiujiang, China. 2Department of Nephrology, The Second Affiliated Hospital of Guangzhou Medical University, Guangzhou, China. ${ }^{3}$ Department of Nephrology, Zhujiang Hospital of Southern Medical University, Guangzhou, China. ${ }^{4}$ Department of Nephrology, Affiliated Sixth People's Hospital, Shanghai Jiao Tong University, No.600, Yi Shan Road, Shanghai, China. ${ }^{5}$ Department of Nephrology, The First Affiliated Hospital of Nanchang University, No. 17, Yongwaizheng Street, Donghu District, Nanchang City 330006, Jiangxi province, China.

Received: 31 December 2019 Accepted: 4 May 2020

Published online: 01 June 2020

\section{References}

1. Johnson DW, Dent H, Hawley CM, McDonald SP, Rosman JB, Brown FG, Bannister K, Wiggins KJ. Association of dialysis modality and cardiovascular mortality in incident dialysis patients. Clin J Am Soc Nephrol. 2009;4(10): 1620-8.

2. Mehrotra R, Devuyst O, Davies SJ, Johnson DW. The current state of peritoneal Dialysis. J Am Soc Nephrol. 2016;27(11):3238-52.

3. Kennedy R, Case C, Fathi R, Johnson D, Isbel N, Marwick TH. Does renal failure cause an atherosclerotic milieu in patients with end-stage renal disease? Am J Med. 2001;110(3):198-204.

4. Isbel NM, Haluska B, Johnson DW, Beller E, Hawley C, Marwick TH. Increased targeting of cardiovascular risk factors in patients with chronic kidney disease does not improve atheroma burden or cardiovascular function. Am Heart J. 2006;151(3):745-53.

5. Zoccali C. Traditional and emerging cardiovascular and renal risk factors: an epidemiologic perspective. Kidney Int. 2006;70(1):26-33.

6. Kaisar M, Isbel N, Johnson DW. Cardiovascular disease in patients with chronic kidney disease. A clinical review. Minerva Urol Nefrol. 2007;59(3): 281-97.

7. Kaisar MO, Isbel NM, Johnson DW. Recent clinical trials of pharmacologic cardiovascular interventions in patients with chronic kidney disease. Rev Recent Clin Trials. 2008;3(2):79-88.

8. Yokoyama M, Watanabe T, Otaki Y, Takahashi H, Arimoto T, Shishido T, Miyamoto T, Konta T, Shibata Y, Daimon M, et al. Association of the Aspartate Aminotransferase to alanine aminotransferase ratio with BNP level and cardiovascular mortality in the general population: the Yamagata study 10-year follow-up. Dis Markers. 2016;2016:4857917.

9. Fuhrmann V, Jager B, Zubkova A, Drolz A. Hypoxic hepatitis - epidemiology, pathophysiology and clinical management. Wien Klin Wochenschr. 2010; 122(5-6):129-39.

10. Henrion J. Hypoxic hepatitis. Liver Int. 2012;32(7):1039-52. 
11. Samsky MD, Patel CB, DeWald TA, Smith AD, Felker GM, Rogers JG, Hernandez AF. Cardiohepatic interactions in heart failure: an overview and clinical implications. J Am Coll Cardiol. 2013;61(24):2397-405.

12. Lofthus DM, Stevens SR, Armstrong PW, Granger CB, Mahaffey KW. Pattern of liver enzyme elevations in acute ST-elevation myocardial infarction. Coron Artery Dis. 2012;23(1):22-30.

13. Weng SF, Kai J, Guha IN, Qureshi N. The value of aspartate aminotransferase and alanine aminotransferase in cardiovascular disease risk assessment. Open Heart. 2015;2(1):e000272.

14. Sette $L H$, Almeida Lopes EP. Liver enzymes serum levels in patients with chronic kidney disease on hemodialysis: a comprehensive review. Clinics (Sao Paulo). 2014;69(4):271-8.

15. Liberato IR, Lopes EP, Cavalcante MA, Pinto TC, Moura IF, Loureiro Junior L. Liver enzymes in patients with chronic kidney disease undergoing peritoneal dialysis and hemodialysis. Clinics (Sao Paulo). 2012;67(2):131-4.

16. Alonso A, Misialek JR, Amiin MA, Hoogeveen RC, Chen LY, Agarwal SK, Loehr LR, Soliman EZ, Selvin E. Circulating levels of liver enzymes and incidence of atrial fibrillation: the atherosclerosis risk in communities cohort. Heart. 2014;100(19):1511-6

17. Torruellas C, French SW, Medici V. Diagnosis of alcoholic liver disease. World J Gastroenterol. 2014;20(33):11684-99.

18. Wu J, Lei G, Wang X, Tang Y, Cheng H, Jian G, Wu X, Wang N Asymptomatic hyperuricemia and coronary artery disease in elderly patients without comorbidities. Oncotarget. 2017;8(46):80688-99.

19. Nyblom H, Berggren U, Balldin J, Olsson R. High AST/ALT ratio may indicate advanced alcoholic liver disease rather than heavy drinking. Alcohol Alcohol. 2004;39(4):336-9.

20. Nyblom $\mathrm{H}$, Nordlinder $\mathrm{H}$, Olsson R. High aspartate to alanine aminotransferase ratio is an indicator of cirrhosis and poor outcome in patients with primary sclerosing cholangitis. Liver Int. 2007;27(5):694-9.

21. Ioannou GN, Boyko EJ, Lee SP. The prevalence and predictors of elevated serum aminotransferase activity in the United States in 1999-2002. Am J Gastroenterol. 2006:101(1):76-82.

22. Charlson ME, Pompei P, Ales KL, MacKenzie CR. A new method of classifying prognostic comorbidity in longitudinal studies: development and validation. J Chronic Dis. 1987;40(5):373-83.

23. Zhang L, Wang F, Wang L, Wang W, Liu B, Liu J, Chen M, He Q, Liao Y, Yu X, et al. Prevalence of chronic kidney disease in China: a cross-sectional survey. Lancet. 2012;379(9818):815-22.

24. Kamimoto Y, Horiuchi S, Tanase S, Morino Y. Plasma clearance of intravenously injected aspartate aminotransferase isozymes: evidence for preferential uptake by sinusoidal liver cells. Hepatology. 1985;5(3):367-75.

25. Zoppini G, Cacciatori V, Negri C, Stoico V, Lippi G, Targher G, Bonora E. The aspartate aminotransferase-to-alanine aminotransferase ratio predicts allcause and cardiovascular mortality in patients with type 2 diabetes. Medicine (Baltimore). 2016;95(43):e4821.

26. Le Couteur DG, Blyth FM, Creasey HM, Handelsman DJ, Naganathan V, Sambrook PN, Seibel MJ, Waite LM, Cumming RG. The association of alanine transaminase with aging, frailty, and mortality. J Gerontol A Biol Sci Med Sci. 2010;65(7):712-7.

27. Liu Z, Que S, Xu J, Peng T. Alanine aminotransferase-old biomarker and new concept: a review. Int J Med Sci. 2014;11(9):925-35.

\section{Publisher's Note}

Springer Nature remains neutral with regard to jurisdictional claims in published maps and institutional affiliations.

Ready to submit your research? Choose BMC and benefit from:

- fast, convenient online submission

- thorough peer review by experienced researchers in your field

- rapid publication on acceptance

- support for research data, including large and complex data types

- gold Open Access which fosters wider collaboration and increased citations

- maximum visibility for your research: over $100 \mathrm{M}$ website views per year

At $\mathrm{BMC}$, research is always in progress.

Learn more biomedcentral.com/submissions 